\title{
Properties of medium density fibreboard (MDF) from kenaf (Hibiscus cannabinus L.) core as function of refining conditions
}

\begin{abstract}
The objective of this study was to evaluate physical and mechanical properties of medium density fibreboard (MDF) panels made from kenaf core as function of fibre geometry and refining conditions. Raw material was prepared by using pressure levels of 3,5 and 7 bar at two heating times, namely 3 and $5 \mathrm{~min}$. The length and width of the fibres were determined employing image analyser. Experimental samples with a target density of $700 \mathrm{~kg}$ mi 3 were produced with $12 \%$ of urea formaldehyde as a binder. Physical properties such as swelling in thickness (TS) and water absorption (WA) of the panels in addition to their mechanical properties including modulus of rupture (MOR), modulus of elasticity (MOE) and internal bonding (IB) were evaluated based on MS 1787:2005. Based on the test results, low digestion pressure produced longer fibre length and panels made from these fibres had higher TS with MOR and MOE than those of the others panels. However, the IB properties of samples were low. Panels made from shorter fibre resulted in contradict properties found above. The ideal properties of the samples were found for the panels made having fibre length of $0.81 \mathrm{~mm}$ and aspect ratio of 23.4. Such sample had 14.6\%, 63.2\%, 30.3 MPa, 3619 MPa and 0.66 MPa for TS, WA, MOR, MOE and IB, respectively.
\end{abstract}

Keyword: A. Fibres; B. Mechanical properties; B. Physical properties; B. Thermomechanical 